\title{
Degradability of polylactide films by commercial microbiological prepara- tions for household composters
}

\author{
Magda Morawska*, Katarzyna Krasowska \\ Gdynia Maritime University, Department of Chemistry and Industrial Commodity Science, Morska Str. 83, 81-225, \\ Gdynia, Poland \\ "Corresponding author: e-mail: m.morawska@wpit.am.gdynia.pl
}

\begin{abstract}
Environmentally friendly polymers such as polylactide are increasingly becoming available for use in packaging applications. The main advantages of polylactide packaging are evident. Polylactide is based on renewable resources and can be degraded in compost or soil. The studies on degradability of polylactide (PLA) films by commercial preparation of mixture of multi-active saprophytic soil microorganisms, bacteria, actinomycetes and fungi have been done. Unmodified PLA film, metalized co-extruded PLA film and modified by silicon oxide PLA film were incubated in the liquid nutritious medium (TSB) prepared to support the growth of microorganisms. The degradability of polylactide films was examined by macro and microscopic observations of surface, changes of mass and crystallinity of polymer samples before and after incubation. The obtained results indicate that the degradation of polylactide was accelerated by the presence of a biological vaccine. It was found that PLA degradation in the inoculated TSB broth was a result of both: enzymatic and chemical hydrolysis.
\end{abstract}

Keywords: biodegradable polymers, polylactide (PLA), enzymatic degradation, DSC.

\section{INTRODUCTION}

The environmental protection, the gradual depletion of the oil resources, as well as technical and economic problems associated with the recycling of packaging wastes, have led scientists to search for new materials for the production of biodegradable packaging. The studies have mainly focused on the development of production technology of compostable, biodegradable polymers, based on renewable raw materials.

Production of biodegradable and biobased polymers is developing rapidly. World production of bioplastics are predicted to grow from 4.16 million as in 2016 to approximately 6.11 million tonnes by $2021^{1,2}$. However, it is still a small percentage of all produced polymers.

It is known, that polylactide is one of the most important biodegradable polymer, whose monomer unit is derived from fermentation of renewable resources. The polylactide is produced in the process of polymerization of the lactic acid obtained from the fermentation of dextrose (derived from corn, potato and others agricultural $)^{3}$. Synthetized polymer reveals properties comparable to those represented by conventional thermoplastic materials. Therefore, polylactide materials play a key role in wide range of applications including packaging, agriculture and biomedical fields ${ }^{4}$. Advantages arising from the use of polylactide packaging are evident, they are obtained from renewable resources and can be degraded in compost or soil, but in specific conditions of temperature and humidity.

Literature includes numerous reports on degradation of this polyester, depending on both physical-chemical and microbiological factors ${ }^{5,6}$. Degradability of PLA by different types of microorganisms has been investigated in various papers. The degradation of PLA by the filamentous fungi ${ }^{5}$, biofilm formation by the different types of microorganisms on the surface of polylactide films ${ }^{7}$ has been investigated. Torres et al. studied different types of microorganisms, which are supposed to be able to use PLA polymers as a source of carbon and energy ${ }^{8}$. Degradation under composting conditions was demonstrated by Sikorska et al. ${ }^{9}$. The authors proved that hydrolytic degradation products of PLA were assimilated by compost microorganisms.

In this connections, microorganisms are the most promising candidates for a possible utilizations of polylactide in environment. So far, polylactide materials were degraded in industrial composting facilities in specific and controlled conditions. The degradation process occurred in modern prisms or in controlled conditions in soil or compost $t^{10,11,12}$. It is interesting to know if the same process of composting of biodegradable plastics can become with equal efficacy in a household as in industrial composters.

The aim of this paper was to investigate if the degradation process of three different types of polylactide films occurs using commercial biological vaccines for household composters. The vaccine is a mixture of multi-active saprophytic soil microorganisms, bacteria, actinomycetes and fungi. These investigations are the preliminary studies on the use of commercial microbiological preparations to biodegrade bioplastics in conditions of household composters.

\section{MATERIAL AND METHODS}

\section{Material}

Three types of commercial packaging films were selected for the tests:

- unmodified co-extruded polylactide film (PLA), with the trade name "BIO 521", which consists of three layers as follows: sealable PLA, core PLA and sealable PLA, with the thickness $40 \mu \mathrm{m}, M_{n}=26600$;

- metalized co-extruded polylactide film (PLA/ALU), with the trade name "BIO 130", which has the following four layers (starting from the external one): vacuum deposited 
aluminium layer, metal-receptive PLA, core PLA and sealable PLA, with the thickness $40 \mu \mathrm{m}, \mathrm{M}_{\mathrm{n}}=26600$; - poly(lactide) film coated with $\mathrm{SiO}_{\mathrm{x}}$ layer $\left(\mathrm{PLA} / \mathrm{SiO}_{\mathrm{x}}\right)$, with the thickness $20 \mu \mathrm{m}$ Alcan Packaging, Belgium, $\mathrm{M}_{\mathrm{n}}=20$ 400;

Raw material was manufactured from NatureWorks ${ }^{\circledR}$ PLA (polylactide), a renewable polymer derived from corn starch. Samples of $20 \times 20 \mathrm{~mm}$ pieces with an average mass of $0.02 \mathrm{~g}$ (PLA, PLA/ALU) and $0.01 \mathrm{~g}\left(\mathrm{PLA} / \mathrm{SiO}_{\mathrm{x}}\right)$ were used. Three replicates of each sample were run.

\section{Degradation experiment}

The degradation of unmodified and physically modified PLA films took place in the a nutritious medium that will support the growth of a wide variety of microorganisms, especially common aerobic and facultative anaerobic bacteria.

Tryptic soy broth (TSB, obtained from Biomaxima) was selected as liquid medium for degradation of polylactide films.

Tryptic soy broth was composed of bacto tryptone (pancreatic digest of casein) - $17.0 \mathrm{~g}$, bacto soytone (peptic digest of soybean meal) $-3.0 \mathrm{~g}$, dextrose $-2.5 \mathrm{~g}$, sodium chloride $-5.0 \mathrm{~g}$, dipotassium hydrogen phosphate $-2.5 \mathrm{~g}$ (per litter purified water). The initial $\mathrm{pH}$ was $7.2 \pm 0.3$. TSB is a nutritious medium that will support the growth of a wide variety of microorganisms, especially common aerobic and facultative anaerobic bacteria. Polylactide samples were immersed in TSB broth with and without addition of biological agent (a commercial formulation named „HUMOBAK”, containing mixture of the selected saprophytic soil microorganisms such as fungi, bacteria and actinomycetes) during 8 weeks. Tubes with $10 \mathrm{ml}$ of broth were stored in the dark and in non-sterile conditions at $37^{\circ} \mathrm{C}$. Maintained not sterile conditions in order to replicate the most natural conditions, where the sample may be infected with other microorganism. However, according to the producer of these preparation, its use decrease the content of pathogenic microflora ${ }^{13}$. The temperature was chosen to create optimal conditions for the growth of microorganisms.

Three film samples were taken out from the tubs after 2, 4, 6 and 8 weeks of composting. Films were washed with distilled water, dried and subjected to further investigation. The degradation of the polylactide films was monitored by macroscopic and microscopic observations of surface structure changes, mass changes and differential scanning calorimetry (DSC).

\section{Preliminary macroscopic and microscopic observations of polylactide films surface}

The test was performed using an optical microscope ALPHAPHOT-2YS2-H Nikon camera equipped with Casio QV-2900UX. PLA samples were observed with and without polarizer. The pictures were taken before and after incubation in selected media.

The mass changes $(\%)$ of degraded polylactide packaging films were performed gravimetrically using an electronic balance Gibertini E 42s. After composting, films were taken out from TSB broth, washed with distilled water and dried at vacuum oven to constant mass. The mass of clean and dried samples of polylactide films after incubation in the broth was compared with those before incubation and the mass changes were calculated. According to the following equation:

$x[\%]=\frac{W-W_{1}}{W} \cdot 100$

where:

$\mathrm{x}$ - changes of mass $\%$,

$\mathrm{W}$ - mass of PLA sample before incubation $\mathrm{g}$,

$\mathrm{W}_{1}$ - mass of PLA sample after incubation $\mathrm{g}$.

Thermal analyses of PLA films were performed on a Differential Scanning Calorimeter Setaram Labsys TG-DTA/DSC with $800^{\circ} \mathrm{C}$ rod. Characteristic of polylactide films thermal properties included melting temperature, melting enthalpy expressed in $\mathrm{J} / \mathrm{g}$ and glass transition temperature expressed in ${ }^{\circ} \mathrm{C}$. The heating rate for the runs was $10^{\circ} \mathrm{C} / \mathrm{min}$ in pure nitrogen atmosphere. The film samples, weighing $0.020 \mathrm{~g}$ were heated in aluminium cells within temperatures from 20 to $200^{\circ} \mathrm{C}$. Empty aluminium cell was used as a reference. Calibration of apparatus was performed using an indium sample. The crystallinity of the polylactide was calculated according to the equation ${ }^{11}$ :

$x_{c}=\frac{\Delta H_{m}}{\Delta H_{m(100 \% P L A)}} \cdot 100 \%$

$X_{c} \quad-$ crystallinity $\%$,

$\Delta H_{m} \quad-$ melting enthalpy $\mathrm{J} / \mathrm{g}$,

$\Delta H_{m(100 \% \text { PLA })} \quad$ - melting enthalpy of $100 \%$ polylactide $93.7 \mathrm{~J} / \mathrm{g}$

\section{RESULTS AND DISCUSSION}

Degradation of polylactide films in TSB broth was evaluated visually at first. The Figures 1 and 2 represents the images of the surface at macro scale of PLA samples before and after incubation in broth and in inoculated broth. Polylactide films after contact with inoculated

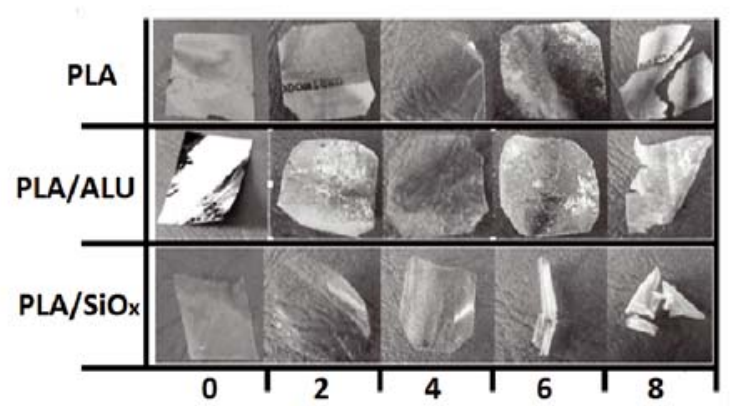

Figure 1. Macroscopic changes of PLA, PLA/ALU and PLA/ $\mathrm{SiO}_{\mathrm{x}}$ samples after $0,2,4,6,8$ weeks of incubation in TSB broth without biological vaccine

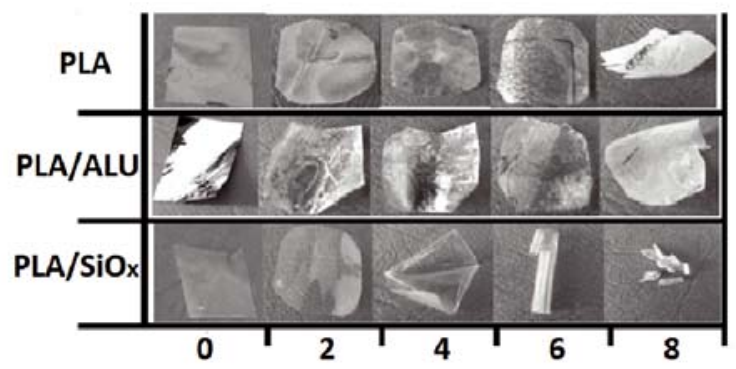

Figure 2. Macroscopic changes of PLA, PLA/ALU and PLA/ $\mathrm{SiO}_{\mathrm{x}}$ samples after $0,2,4,6,8$ weeks of incubation in TSB broth with biological vaccine 
broth were more damaged compered to samples after incubation in pure broth. During the incubation time, colour and glossy of the films changed significantly. PLA films and PLA/ALU after two weeks of immersion in vaccinated broth, lost their transparency and covered with product of the metabolic activity of the microorganisms (white and brown points on the films surface). The changes in colour and tarnishing of the surface could be caused by crystallinity changes, as was demonstrated in the following thermal analysis. PLA/ $\mathrm{SiO}_{\mathrm{x}}$ samples were initially transparent but after 6 weeks of incubation they have become opaque, fragile and began to disintegrate - particularly in the broth with microbiological preparation. Disintegration in pure broth was observed after 8 weeks of incubation.

Microscopic studies confirmed preliminary macroscopic observations. The micrographs of the PLA films surfaces before and after 8 weeks incubation in TSB with and without biological vaccine are shown in Figure 3.

The microscopic photographs of PLA samples incubated in TSB broth with and without vaccine show the changes of the surface, which were visibly rough in comparison to smooth surface of the control samples. PLA and PLA/ALU films after contact with inoculated broth were discolored which may indicate on microbial activity. After 8 weeks of incubation with microbiological vaccine some dark places were observed. This phenomena might be an evidence of presence of the microorganism agglomerations and discoloration of material caused by microbial metabolites. Microscopic observation of the polylactide surface changes suggest the enzymatic degradation of the polymer. It is known that the polymeric materials subjected to enzymatic hydrolysis has a rough surface and erosion patterns. While chemical hydrolysis occurs primarily inside polymer, where the surface re- mains intact ${ }^{14,15}$. On the surface of $\mathrm{PLA} / \mathrm{SiO}_{\mathrm{x}}$ sample, after incubation in broth without vaccine, the cracking of surface was observed. The cracks were probably caused by only mechanical damage of $\mathrm{SiO}_{\mathrm{x}}$ layer. Typical changes of microorganisms acting on $\mathrm{PLA} / \mathrm{SIO}_{\mathrm{x}}$ surface were not observed. After degradation in inoculated TSB broth, the distinctly fragmentation of $\mathrm{PLA} / \mathrm{SiO}_{\mathrm{x}}$ were observed and it was impossible to take micrographs of this sample.

Susceptibility of PLA samples to degradation in pure broth and in inoculated broth was also evaluated by changes of polylactide mass after incubation. The results of the mass changes of the unmodified and modified polylactide films after incubation are presented in Figure 4 and Figure 5.

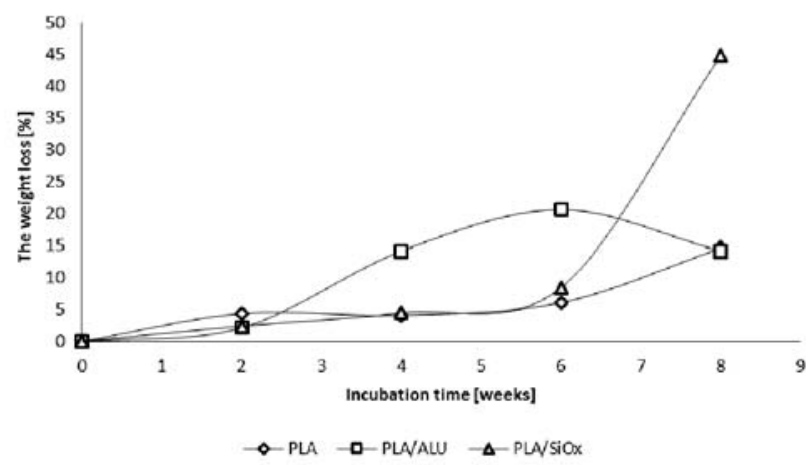

Figure 4. The mass loss of the polylactide films [\%] after incubation to the TSB broth without biological vaccine

The mass loss of the investigated polylactides depended on the incubation time of samples in TSB broth. The greatest mass loss (about 35, 28, 90\% respectively) was observed for the PLA, PLA/ALU and PLA/SiO ${ }_{x}$ films after contact with inoculated broth. The mass loss of films immersed in unvaccinated broth was significantly

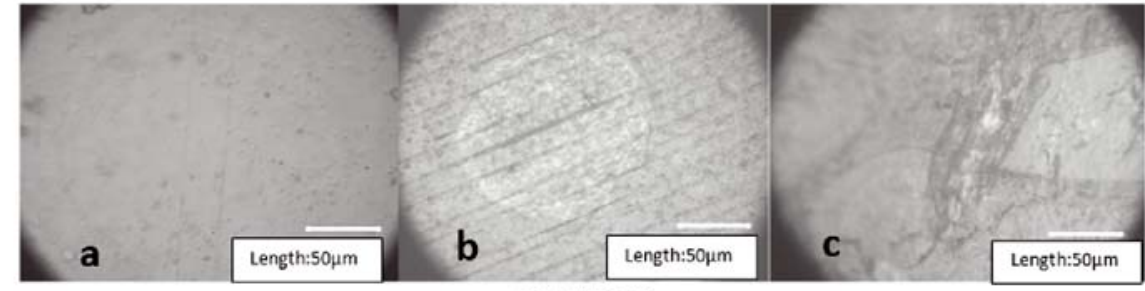

PLA/ALU

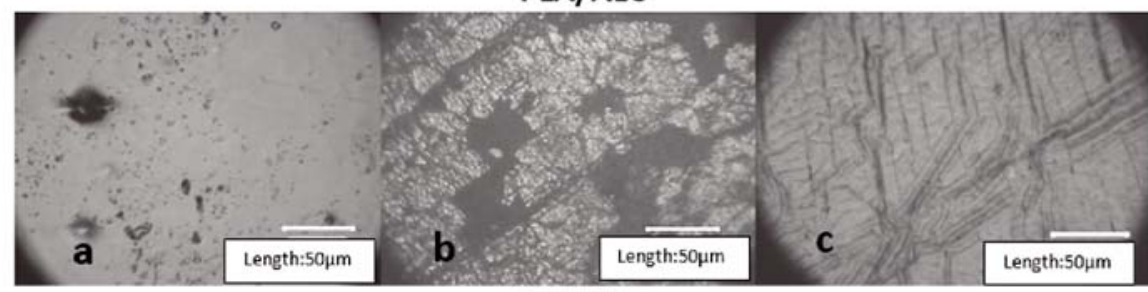

$\mathrm{PLA} / \mathrm{SiO}_{\mathrm{x}}$

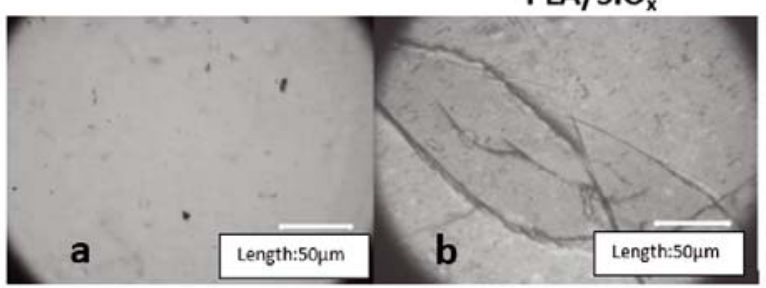

Insufficient sample for microscopic analysis

Figure 3. The photomicrographs of the surfaces of the polylactide films: (a) before incubation and after 8 weeks of incubation in (b) TSB broth without and (c) with biological vaccine 
lower. During the 6 weeks of incubation, PLA/ALU samples lost aluminum layer and the higher mass losses were observed. The lower mass losses of PLA/ALU film at 8 week were probably associated with water absorption of material. Mass changes of $\mathrm{PLA} / \mathrm{SiO}_{\mathrm{x}}$ indicated on only slight decrease (up to approximately $10 \%$ of the initial mass of the sample) after 6 weeks of incubation in compost. After 8 weeks, the mass loss strongly increased. $\mathrm{PLA} / \mathrm{SiO}_{\mathrm{x}}$ film immersed in inoculated TSB broth lost approximately $90 \%$ of initial mass after 8 weeks of incubation (Fig. 5). The higher degradation of this material may be explained by its lower thickness and consequently easier penetration of water into the matrix of polymer.

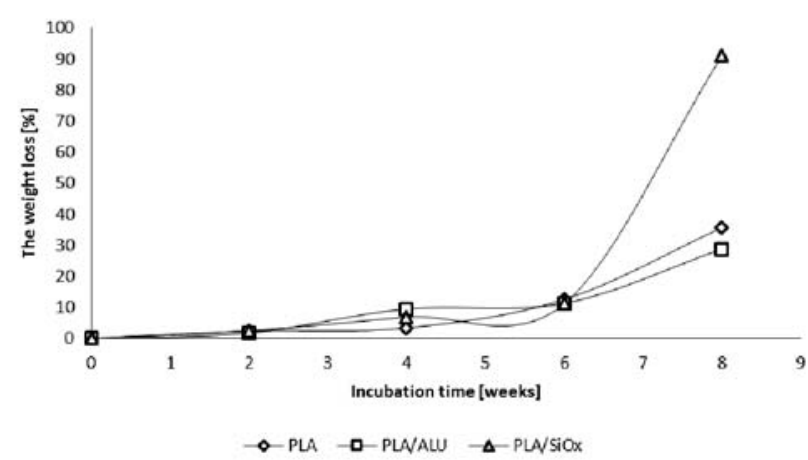

Figure 5. The weight loss of the polylactide films [\%] after incubation to the TSB broth with biological vaccine

It is known, that degradation of polylactide in aqueous media occurs in two steps. The first step involves - the penetration of water into the polymer and the hydrolysis of ester bonds within the matrix of polymer. Degradation occurs in the bulk, so soluble oligomers are generated in polymer matrix. If the polymer samples are thin, polymer chains with soluble molecular mass can readily diffuse out from samples. The mass of polymer remains less or more constant. In the next stage, the oligomers are dissolved in aqueous medium, therefore the mass of polymer decreases ${ }^{16,17}$. At this stage, soluble oligomers and the lactic acid can be metabolized or mineralized by microorganisms presents in environment ${ }^{18}$.

The biodegradability of polylactide (PLA) films has been characterized by means of Differential Scanning Calorimetry (DSC). The morphology of a polymeric material (amorphousness or semicrystallinity) plays a critical role in the degradation process. DSC studies revealed the significant thermal properties of the samples, such as glass transition temperature $(T g)$ and melting temperature (Tm). $\mathrm{Tg}$ and $\mathrm{Tm}$ correspond to the amorphous and crystalline regions of the materials, respectively. Tested polylactide films are semicrystalline polymers and the major features of their DSC curve are the $T g$ at $58^{\circ} \mathrm{C}$ and its melting peak $(\mathrm{Tm})$ at about $150^{\circ} \mathrm{C}$. The degradation of polymers occurs at higher rate in the amorphous regions, causing the increase of the overall material crystallinity. The morphology of polymers is a crucial factor which has an impact on biodegradability. The amorphous parts of polymer, due to their loose packed structure, are more accessible for microorganisms. The crystalline regions of the polymer are more resistant to degradation. The biodegradability of PLA decreases with increasing of the crystallinity degree ${ }^{19}$. DSC results confirmed that the loss of sample transparency is due to the crystallization process. Increase of polylactide crystallinity was observed for samples degraded in TSB broth and TSB broth with vaccine (Table 1). However, the degradation process of all tested PLA films was much faster in broth with Humobak vaccine. It was confirmed previously by macroscopic and microscopic observation of polymer samples after incubation. Decrease of $T g$ for all tested polylactide films after 8 weeks of degradation process in broth was observed. Decrease of $T g$ could be attributed to the plasticizing effect, probably associated with the reduction of the molecular mass of polymers. The same correlation was observed by Musioł et al. examining degradation of thermoformed rigid polylactide. The author recorded a glass transition temperature decrease after degradation in compost pile ${ }^{20}$.

\section{CONCLUSION}

The presence of the microbiological preparation in TSB broth accelerated the degradation of investigated polylactide films compared to the degradation in TSB broth without microbiological vaccine.

Microscopic observations of the polylactide surface morphology incubated with biological vaccine indicated on the microorganisms activity. The crystallinity degree of tested films increased during degradation process, which resulted in the loss of films transparency. The insignificant mass losses during the initial weeks of incubation might be explained by hydrolytic degradation of ester bonds inside the polymer matrix. The studies revealed, that the mass of all tested polylactide films decreased significantly, after longer incubation time in inoculated broth (8 weeks). The polylactide samples requires a longer incubation time for degradation in aqueous medium with biological vaccine. However, in order to obtain comprehensive information of the polylactide films degradation by commercial microbiological preparations, more detailed analysis ought to be conduc-

Table 1. Thermal analysis of polylactide films after 8 weeks of incubation in TSB broth with and without biological vaccine

\begin{tabular}{|c|c|c|c|}
\hline & $\operatorname{Tg}\left[{ }^{\circ} \mathrm{C}\right]$ & $\Delta \mathrm{H}_{\mathrm{m}}[\mathrm{J} / \mathrm{g}]$ & $\begin{array}{c}\text { Crystallinity } \\
x_{c}[\%]\end{array}$ \\
\hline PLA before incubation & 58.2 & 11.4 & 12.2 \\
\hline PLA incubated in TSB broth & 53.9 & 31.1 & 33.2 \\
\hline PLA/ALU before incubation & 58.4 & 12.9 & 13.8 \\
\hline PLA/ALU incubated in TSB broth & 57.1 & 33.5 & 35.8 \\
\hline PLA/ALU incubated in TSB broth with biological vaccine & 50.9 & 44.1 & 47.0 \\
\hline $\mathrm{PLA} / \mathrm{SiO}_{\mathrm{x}}$ incubated in TSB broth & 50.9 & 52.5 & 56.1 \\
\hline $\mathrm{PLA} / \mathrm{SiO}_{\mathrm{x}}$ incubated in TSB broth with biological vaccine & \multicolumn{3}{|c|}{ Insufficient sample for thermal analysis } \\
\hline
\end{tabular}

* The percent crystallinity was calculated based on the value of $93.7 \mathrm{~J} / \mathrm{g}$ as the heat of melting of fully crystalline polylactide. 
ted. In conclusion, the addition of the microbiological agent to TSB broth accelerated the process of enzymatic degradation of polylactide films. Interesting problem is, whether acceleration of degradation could become with equal efficacy in a household composter with composting microbiological vaccine.

\section{LITERATURE CITED}

1. http://docs.europeanbioplastics.org/2016/publications/ EUBP_Facts_and_Figures_2017.pdf

2. http://www.biotworzywa.com.pl/pl/produkcja-biotworzywrosnie-mimo-niskiej-ceny-ropy-naftowej/

3. Young-Jung, W., Jin-Nam Kim, K. \& Hwa-Won, R. (2006). Biotechnological Production of Lactic Acid and Its Recent Applications. Food Technol. Biotechnol. 44(2), 163-172. Retrieved August 20, 2016, from Food Technology and Biotechnology database on the World Wide Web:http:// /www.ftb.com.hr

4. Dove, A.P. \& Becker, J. (2011). Poly(lactide)s as Robust Renewable Materials. W Mathers R.T. \& Meier, M.A. (ed.), Green Polymerization Methods, 201-220. Wiley-VCH. DOI: 10.1002/9783527636167.ch9.

5. Szumigaj, J., Żakowska, Z., Klimek, L., Rosicka-Kaczmarek, A. \& Bartkowiak, A. (2008). Assessment of Polylactide Foil Degradation as a Result of Filamentous Fungi Activity. Polish J. Environ. Stud. 17(3), 335-341.

6. Krueger, M., Harms, H. \& Schlosser, D. (2015). Prospects for microbiological solutions to environmental pollution with plastics. Appl. Microbiol. Biotechnol. Nov. 99(21); 8857-8874, DOI: 10.1007/s00253-015-6879-4.

7. Walczak, M., Świontek-Brzezińska, M., Sionkowska, A., Michalska, M. \& Jankiewicz, U. (2015). Biofilm formation on the surface of polylactide during its biodegradation in different environments. Coll. Surf. B, 136, 340-345. DOI:10.1016/j. colsurfb.2015.09.036.

8. Torres, A., Li, S., Roussos, S. \& Vert, M. (1996). Degradation of 1- and D,1- lactic acid oligomers in the presence of Fusarium moniliforme and Pseudomonas putida. J. Environ. Polym. Degrad. 4, 213-216. DOI: 10.1007/BF02070690.

9. Sikorska, W., Musioł, M., Nowak, B., Pająk, J. \& Łabużek, S. (2015). Degradability of polylactide and its blend with poly(R,S)-3-hydroxybutyrate in industrial composting and compost extract. Int. Biodet. Biodegr. 101, 32-41. DOI:10.1016/j. ibiod.2015.03.021.

10. Kolstad, J., Vink, E., De Vild, B. \& Debeer, L. (2012). Assessment of anaerobic degradation of Ingeo polylactides under accelerated landfill conditions. Polym. Degrad. Stabil. 97, 1131-1141. DOI:10.1016/j.polymdegradstab.2012.04.003.

11. Karlsson, S., Albertsson, A. \& Hakkarainen, M. (2000). Rapid (bio)degradation of polylactide by mixed culture of compost microorganisms-low molecular mass products and matrix changes. Polymer 41. DOI: 10.1016/S0032-3861(99)00393-6.

12. Kalea, G., Aurasa, R., Singha, S. \& Narayan, R. (2007). Biodegradability of polylactide bottles in real and simulated composting conditions. Polym. Test. 26, 1049-1061. DOI: 10.1016/j.polymertesting.2007.07.006.

13. Zydlik, Z. \& Zydlik, P. (2013). The effect of microbiological products on soil properties in the conditions of replant disease. Zemiderbiste 100(1), 19-24. DOI: 10.13080/z-a.2013.100.003. 14. Sikorska, W. (2000). The influence of morphology on properties and biodegradation of copolymers and polymer blends containing atactic poly(3-hydroxybutyrate) (in Polish). Unpublished PhD doctoral dissertation. Zabrze, Poland.

15. Li, S., Girard, A., Garreau, H. \& Vert, M. (2001). Enzymatic degradation of polylactide stereocopolymers with predominant d-lactyl contents. Polym. Degrad. Stabil. 71, 61-67. DOI: 10.1016/S0141-3910(00)00152-X.

16. Li, S. (1999). Hydrolytic Degradation Characteristics of Aliphatic Polyesters Derived from Lactic and Glycolic Acids. J.
Biomed. Mater. Res. 48(3), 342-353. DOI:10.1002/(SICI)10974636(1999)48:3<342::AID-JBM20>3.0.CO;2-7.

17. Lyu, S. \& Untereker, D. (2009). Degradability of Polymers for Implantable Biomedical Devices. Int. J. Mol. Sci. 10(9), 4033-4065. DOI:10.3390/ijms10094033.

18. Torres, A., Li, S., Roussos, S. \& Vert, M. (1996). Screening of Microorganisms for Biodegradation of Poly(Lactic Acid) and Lactic Acid-Containing Polymers. Appl. Environ. Microbiol. 62, 2393-2397.

19. Tokiwa, Y., Calabia, B., Ugwu, C. \& Aiba, S. (2009). Biodegradability of Plastics. Inter. J. Molec. Sci. 10(9), 3722-3742. DOI:10.3390/ijms10093722.

20. Musioł, M., Sikorska, W., Adamus, G., Janeczek, H. \& Richert, J. (2016). Forensic engineering of advanced polymeric materials. Part III - Biodegradation of thermoformed rigid PLA packaging under industrial composting conditions. Waste Manage 52, 69-76. DOI: 10.1016/j.wasman.2016.04.016. 